beiden Beinen im Beruf stehen. Nur so können aus der Wirklichkeit der beruflichen Pflege heraus praktisch relevante Themen gestaltet werden. Andererseits ist die Zusammenstellung einer Liste sehr anspruchsvoll und verlangt ein gutes Netzwerk mit Kollegen der eigenen Berufsgruppe. In einem Flächenland mit ungleicher Verteilung von beruflich Pflegenden ist das eine besondere Aufgabe.

\section{Aufregung nach Landtagswahl}

Besondere Aufmerksamkeit hat, auch bundesweit, die Landtagswahl in Schles-
wig-Holstein auf sich gezogen. Anders als in Rheinland-Pfalz, dort haben alle Parteien und Landtagsabgeordneten für das Kammergesetz gestimmt, gab es in der neuen Koalition der Landesregierung in Schleswig-Holstein Vorbehalte gegenüber der Pflegeberufekammer. Sie mündeten in einer intensiven Auseinandersetzung darüber, ob die vorgesehene rechtliche Struktur der Kammer in Richtung des bayerischen Modells verändert wird. Letztlich wurde entschieden, dass die vorgesehene Änderung der gesetzlichen Regelungen zur Pflegeberu- fekammer nicht zum Bestandteil der Koalitionsvereinbarung wird. Insofern bleiben für den Errichtungsausschuss alle gesetzlichen Arbeitsgrundlagen bestehen und auch die Kammer selbst kann wie vorgesehen an den Start gehen.

\section{Frank Vilsmeier}

Vorstand, Stellvertretender Vorsitzender des Errichtungsausschusses der Pflegeberufekammer Schleswig-Holstein

\title{
Neuer Berufsmix für ein besseres Leben bei Demenz erforderlich
}

\begin{abstract}
Defizite in der Diagnostik und Versorgung. Pflegen bis zur Erschöpfung: Wer sich intensiv um demente Angehörige kümmert, ist oft am Ende seiner Kräfte. 59\% der im Rahmen des aktuellen Pflegereports 2017 der DAK-Gesundheit Befragten geben dies an. Selbst im weiteren Umfeld von Menschen mit Demenz sagt jeder Dritte, oft erschöpft zu sein.
\end{abstract}

$\mathrm{N}$ eun von zehn pflegenden Angehörigen fordern mehr Unterstützung und nehmen dabei gleichzeitig zu wenig professionelle Hilfe in Anspruch. Außerdem sind $80 \%$ der Deutschen der Meinung, dass Menschen mit Demenz und deren Familien mehr Respekt verdienen.

Der Pflegereport zeigt zudem, dass 35\% der Befragten mit Demenzerfahrung den eigenen Haushalt für den besten Lebensort für Menschen mit Demenz halten, jeder Fünfte sieht dagegen Wohngruppen als die beste Betreuungsform Demenzkranker an. In Wohngruppen leben aktuell jedoch nur knapp 2\% der Betroffenen. Wunsch und Wirklichkeit klaffen hier weit auseinander.

Erarbeitet wurde der DAK-Pflegereport unter Federführung des AGP Institut Sozialforschung an der Evangelischen Hochschule Freiburg. Im Mittelpunkt stand dabei die Frage: „Ist trotz Demenz ein gutes Leben möglich?“.

\section{Anderer Berufsmix nötig}

Das sei grundsätzlich erreichbar, sagte hierzu der zuständige Autor des Reports, Prof. Dr. Thomas Klie, bei der Presse- konferenz der DAK-Gesundheit zur Veröffentlichung des Reports. Jedoch sei dies an einige Bedingungen geknüpft. Benötigt werde unter anderem ein anderer Berufsmix in der Pflege und auch andere Kompetenzen der professionell Pflegenden. Denn, so Klie weiter, „hat 97\% des Tagesablaufs eines an Demenz erkrankten Menschen nichts mit der Pflege und Medizin zu tun. Der größte Bedarf liegt im Bereich der Unterstützung und vor allem in der Teilhabe am Leben. Das müssen wir viel stärker als bisher in den Blick nehmen“. Die dabei zu erbringenden Leistungen würden jedoch „weit über die heutigen Leistungen der in der stationären Pflege eingesetzten zusätzlichen Betreuungskräfte hinausgehen“.

\section{Vollzugsdefizit beim neuen Pflegebedürftigkeitsbegriff}

Ein deutliches Vollzugsdefizit sieht Klie in der Umsetzung des neuen Pflegebedürftigkeitsbegriffes. Überwiegend werde dieser in den Pflegeeinrichtungen nicht umgesetzt. Es fehle die Anpassung des Leistungserbringungsrechts. „Es reicht nicht aus, einen neuen Pflegebedürftigkeitsbegriff zu schaffen und diesen zu feiern, ohne die richtigen Rahmenbedingungen für dessen Umsetzung zu schaffen", betonte Klie.

„Investitionen in das Personal müssen bei der Wertschätzung ansetzen. Fehlt diese, dann fliehen die Mitarbeiter", machte er weiter deutlich. Auf der anderen Seite seien Mitarbeiter „ohne $\mathrm{Hu}$ mor" in der Begleitung von Menschen mit Demenz „fehl am Platz“.

\section{Zu wenig auf professionelle Pflege gesetzt}

Klie wies zudem darauf hin, dass in Deutschland bei der Pflege zu wenig professionelle Kräfte in Anspruch genommen werden. „Wir haben in Deutschland eine hohe Akzeptanz der Pflegeberufe, jedoch ein Nachfrageproblem bei den Dienstleistungen, vor allem in der häuslichen Pflege“, sagte er weiter. Eine der Gründe hierfür könnte darin liegen, dass „die Pflege in Deutschland zu kompliziert aufgebaut ist".

Deutlich wird er auch zur stationären Pflege. „Im internationalen Vergleich ist die Langzeitpflege in Deutschland dramatisch unterfinanziert", mahnte der Pflegewissenschaftler abschließend.

www.dak.de 